\title{
Human cystic echinococcosis in southwest Iran: a 15-year retrospective epidemiological analysis of 501 consecutive hospitalized cases (2004-2018)
}

\section{Reza Shahriarirad}

Shiraz University of Medical Sciences

Amirhossein Erfani

Shiraz University of Medical Sciences

Mehrdad Eskandarisani

Shiraz University of Medical Sciences

Mohammad Rastegarian

Shiraz University of Medical Sciences

Hajar Taghizadeh

Shiraz University of Medical Sciences

Bahador Sarkari ( $\nabla$ sarkarib@sums.ac.ir)

Shiraz University of Medical Sciences https://orcid.org/0000-0003-2045-9057

Research article

Keywords: Cystic echinococcosis, Hospital record, Fars Province, Southwestern Iran

Posted Date: December 3rd, 2019

DOl: https://doi.org/10.21203/rs.2.18051/v1

License: (9) (i) This work is licensed under a Creative Commons Attribution 4.0 International License.

Read Full License 


\section{Abstract}

Background: Cystic echinococcosis (CE) is considered a neglected disease with significant mortality and morbidity in most of the developing countries. The current study aimed to retrospectively assess the epidemiologic and clinical features of human CE in a 15-year period in Fars province, southwestern Iran.

Methods: Hospital records of patients undergone surgeries for CE in two main hospitals in Fars Province, southwestern Iran in a 15-year period (2004-2018) were reviewed and data concerning the patients' demographical and clinical features were retrieved from each record.

Results: A total of $501 \mathrm{CE}$ surgical cases were recorded during a 15-year period, giving an average annual incidence of 33.4 cases in which 242 (48.6\%) were male and 256 (52.4\%) were female. Patients' age ranged from 2 to 96 years, with a mean age of $34.92( \pm 19.87)$ years. A significantly higher rate of CE cases was noted in subjects $\geq 50$ years of age. The highest frequency of cases (62: $12.5 \%$ ) was recorded in the year 2017. The most commonly involved organs were liver (339 cases; $57.8 \%$ ), and lung (279 cases; $47.6 \%)$. Concurrent involvement of two organs was seen in $58(9.9 \%)$ cases of both lung and liver, $10(1.6 \%)$ cases of lung and other locations (but not liver) and $23(3.9 \%)$ cases of liver and other locations (but not lung). Recurrence was noted in 67 (13.37\%) of the cases. The size of the lung hydatid cyst varied, ranging between 2 to $24 \mathrm{~cm}$ (mean $=7.33, S D=3.737$ ). The size of liver hydatid cysts ranged from 1 to $26 \mathrm{~cm}$ (mean: 9.04, SD= 4.275).

Conclusion: The findings of the current study demonstrated a nearly constant prevalence of CE during the last 15 years in southern Iran. Further studies are needed to find out the reasons behind the recurrence of the disease, which is substantial, in surgically-treated patients.

\section{Background}

Cystic echinococcosis (CE), due to Echinococcus granulosus is one of the most significant zoonotic diseases, throughout most parts of the world [1]. Herbivores such as sheep, goats, and swine are intermediate hosts of $E$. granulosus, which usually become infected through eating the worm eggs, passed in the carnivores' feces. Carnivores, as definitive hosts, ingest the cyst-containing organs of herbivores and harbor worms in their digestive system. Humans become infected via ingesting embryonated eggs through hands, water, or food, contaminated with parasite eggs, passed through the feces of definitive hosts, mainly dogs. The larvae spread throughout the blood and lymphatic circulation and transport to the lungs, liver, and other organs where it becomes hydatid cyst.

CE is asymptomatic at the early phases of the infection; however, it becomes symptomatic when the cysts become larger or complicated. Clinical manifestations of CE vary and depend on the size, location, and condition of the cystic structure.

CE is considered a neglected disease with significant mortality and morbidity in most of the developing countries [1]. The disease is a major health and economic challenge in the Middle East countries, 
including Iran where about $1 \%$ of all hospital surgeries are accounted for this disease [1-5]. The overall prevalence of CE in Iran is reported to be $4.2 \%$ with the highest prevalence in the South area with $5.8 \%$ and the least in the central area with $2.2 \%$ of prevalence rate [6].

$\mathrm{CE}$ is more frequently occurring in rural and nomadic communities where people have continuous contact with dogs, the definitive host of E. granulosus. Studies in both West and East Azerbaijan and Hamedan provinces in Iran demonstrated that ruralresidents constitute the most common referral cases of CE [79].

$\mathrm{CE}$ is one of the most important parasitic diseases in Fars province in the southwest of Iran. Fars province is the center of agriculture and animal husbandry in Iran and one of the most important and populated tribal nomads (Qashqai) resides in this area. The burden and incidence of CE which are the basis for the development of appropriate control programs are poorly known in Fars province, in southern Iran. Hence, the current study was designed and conducted to assess the epidemiologic and clinical features of human CE in a 15-year period in Fars province, southwestern Iran, based on the hospital records.

\section{Methods}

\section{Study Area}

This study was conducted in Fars Province in southern Iran. The district is located at an altitude of 1545 $m$ above the sea level at geographical coordinates of $29^{\circ} 36^{\prime} 37^{\prime \prime} \mathrm{N}$ latitude and $52^{\circ} 31^{\prime} 52^{\prime \prime}$ E longitudes. The area has a hot summer and moderate winter. Agriculture has always been a major part of the economy in Fars province.

\section{Data Collection}

In this retrospective study, hospital records of CE patients were reviewed and data were retrieved for a 15year period, from 2004 to 2018 at the main university-affiliated and referral hospitals (Nemazi and Shahid Faghihi) in Shiraz, capital of Fars Province. The hospital records for a few years of the study were not available in the hospital database. Therefore, the medical records were searched manually by members of the research team which consisted of physicians as well as medical students. From each patient's record, the demographic, epidemiological and clinical data including age, sex, residence area, cyst location and characteristics, duration of hospitalization and history of relapse were extracted. Definitive CE diagnosis was mainly based on histological examinations of the cysts, removed from the patient during or after the surgery.

\section{Statistical analysis}


Collected data were analyzed by SPSS Ver. 22 (SPSS Inc., Chicago, IL, USA). Data of patients who underwent surgery due to hydatid cyst were tabulated according to sex, age, residence area, cyst features, and also other demographic and clinical features. Chi-square was used to determine the association between $\mathrm{CE}$ and patients' demographics or clinical values.

\section{Results}

A total of 501 CE surgical cases were diagnosed and underwent surgery in two main hospitals in the 15-year period from 2004 to 2018, giving an average annual incidence of 33.4 cases. The mean age of the patients was $34.92( \pm 19.87)$ years. The youngest patient was a 2 years old girl and the oldest one was a 96 years old man. The majority of the cases (26.4\%) were $\geq 50$ years old. Out of 501 CE cases, 242 (48.6\%) were male while 256 (51.4\%) were female. The highest frequency of cases (62: 12.5\%) was recorded in the year 2017. Figure 1 shows the frequency of CE cases, per year.

Considering the residential areas of the patients, the majority of cases (76\%) originating from Fars Province, whereas cases from neighboring Provinces mainly Kohgiluyeh and Boyer-Ahmad (7\%) and Bushehr (4.4\%), were also among the patients.

The most commonly involved organs were liver (339 cases; 57.8\%), and lung (279 cases; (47.6\%). Also, 46 (7.8\%) cases had hydatid cyst in other locations including spleen, heart, diaphragm, etc. Concurrent involvement of two organs was seen in 58 (9.9\%) cases of both lung and liver, 10 (1.7\%) cases of lung and other locations (excluding liver) and 22 (3.8\%) cases of liver and other locations (excluding lung). Table 1 shows the location of hydatid cyst in CE cases in southwest Iran.

Table 1 location of hydatid cysts in CE cases in southwestern Iran, based on hospital record, during a 15-year period (2004-2018) 


\section{Frequency (No.) Percent (\%)}

Location

\begin{tabular}{lcc}
\hline Lung & 279 & 47.6 \\
Liver & 339 & 57.8 \\
Spleen & 14 & 2.4 \\
Pelvic cavity & 7 & 1.2 \\
Heart & 4 & 0.7 \\
Sub diaphragm & 5 & 0.9 \\
Abdominal and peritoneal cavity & 5 & 0.9 \\
Other locations, ** & 12 & 2 \\
Lung and liver & 58 & 9.9 \\
Lung and Spleen & 2 & 0.3 \\
Lung and Pelvic cavity & 1 & 0.2 \\
Lung and sub diaphragm & 2 & 0.3 \\
Lung and Heart & 2 & 0.3 \\
Lung and other locations & 3 & 0.5 \\
Liver and Spleen & 3 & 1 \\
Liver and pelvic cavity & 6 & 1 \\
Liver and sub diaphragm & 3 & 0.5 \\
Liver and abdominal and peritoneal cavity & 3 & 0.5 \\
Liver and other locations** & 5 & 0.9 \\
Spleen and pelvic cavity & 1 & 0.2 \\
\hline
\end{tabular}

* CBD, mediastina, pancreas

** Kidney, mediastinum, mesothelium of the terminal ileum, pancreas, psoas muscle

Multiple organ involvement of lung, liver, and spleen were seen in $2(0.3 \%)$ cases, lung, liver and pelvic cavity in $1(0.2 \%)$ case, lung liver and sub-diaphragm in $1(0.2 \%)$ case, and lung liver and pancreas in $1(0.2 \%)$ case.

Secondary hydatid cyst or relapse were seen in $67(13.37 \%)$ of the patients, in which the secondary cysts in 28 (41.8\%) were lung cysts, 42 (62.7\%) were liver cyst, 1 (1.5\%) was pelvic cyst, 1 (1.5\%) was spleen cyst, 1 (1.5\%) was abdominal and peritoneal cavity and 1 (1.5\%) was in the mesothelium of the terminal ileum. It is worth mentioning that 11 of the cases underwent surgery due to tertiary hydatid cyst or relapse for the third time and also 
3 of the cases underwent a fourth surgery due to hydatid cyst. Table 2 shows the demographic features of the CE patients along with the cyst features.

From the 279 cases of lung hydatid cyst, the majority (174: 74\%) had a single cyst while 46 (19.6\%) cases had two and 15 (6.4\%) cases had three or more cysts. It is worth knowing that from the 279 cases of lung hydatid cyst, 157 (56.3\%) cases were male while 120 (43.3\%) were female. There was a significant correlation between lung hydatid cysts and the patients' gender $(\mathrm{P}=0.000)$. The size of the lung hydatid cyst varied, ranging between 2 to $24 \mathrm{~cm}$ (mean $=7.33, \mathrm{SD}=3.737)$ with the highest frequency $(175: 96.2 \%)$ of the largest diameter of the cyst being in group 11 to $15 \mathrm{~cm}$. The lung cyst area ranging from 2 to 490 $\mathrm{cm}^{2}$ (mean $42.27, \mathrm{SD}=50.061$ ) with the majority in under $26 \mathrm{~cm}^{2}$ group (83:48.3\%)

In our study, among the 339 cases of liver hydatid cyst, 194 (68.1\%) had single cysts, 56 (19.6\%) had two cysts, and 35 (12.3\%) had three or more cysts. The size of liver hydatid cysts ranged from 1 to $26 \mathrm{~cm}$ (mean: 9.04, $\mathrm{SD}=4.275$ ) with the highest frequency of the largest diameter of the cyst being in group 6 to $10 \mathrm{~cm}$. The area square centimeters of the liver cyst ranged from 1 to $392 \mathrm{~cm}^{2}$ (mean=58.43, $\left.\mathrm{SD}=52.556\right)$ with the majority (82: $31.9 \%$ ) in under $26 \mathrm{~cm}^{2}$ group.

In other locations, apart from lung and liver, the number of cysts varied were 21 (3.6\%) cases had only a single cyst, $7(1.2 \%)$ cases had two cysts, $8(1.4 \%)$ cases had three or more. The size of the cysts varied, ranging between 2 to $18 \mathrm{~cm}$ (mean=8.73, SD=4.527) with the highest frequency (19: 55.9\%) being in the 6 to $10 \mathrm{~cm}$ group. The area square centimeters of the cysts in other locations ranged between 3 to $314 \mathrm{~cm}^{2}($ mean $=59.14, \mathrm{SD}=$ 64.287).

\section{Discussion}

CE is a zoonotic disease that occurs all over the world and causes substantial public health problems and economic losses in several countries. CE is considered endemic in the entire Mediterranean zone including all countries from the Middle East $[1,10]$. Studies from different parts of Iran confirm the fact that this country is an endemic or even hyperendemic area for CE [5, 10-12]. The incidence rate of CE in 
Iran has been reported to be $1.3-3 / 100,000$ population. A higher rate of incidence $(4.5$ cases $/ 100,000$ /year) has been reported in neighboring Iraq [13].

Cases of CE are being reported from all the 32-Provines of Iran but the disease is more prevalent in sheepbreeding areas, including Fars province in the southwest of the country. The current study describes a detailed status of the geographical distribution and the epidemiological picture of CE in southern Iran through retrospective reviewing of the patients' hospital records during a 15-year period. The annual surgical cases of CE were found to be 33.4 cases. It should be noted that some of the CE patients may seek treatment in local hospitals, where sufficient equipment is available for CE operation. Considering these people, the annual rate of $\mathrm{CE}$ is higher than the current reported rate. These data clearly illustrate the magnitude of the problem of human CE in the south of Iran and provides the basic data for the implementation of surveillance and control programs for CE in the area.

CE is reported to have a higher prevalence rate in women in Iran which can be due to household tasks of women that are traditionally a part of their daily routine life $[7,14]$. In the current study, women were found to be at higher risk of $\mathrm{CE}$ infection than men. This finding is in line with our previous study and also a study by Abdulhameed in Iraq, where females were reported to be the main victim of $C E$, in a retrospective hospital-based study conducted in a neighboring city, Basrah in Iraq $[5,13]$. Previous studies suggested that women have the highest chance of contact with sources of infection such as dogs, soil, vegetable, etc. $[5,15,16]$. Housewives, especially in rural areas, in which the most infected cases can be found, have the highest chance of contact with the sources of infection. Contact with contaminated vegetables, cleaning the house containing the dog feces, desire to eat soil (Geophagy) as a longing in pregnant embrace the etiological matters. However, in studies conducted by Cohen et al. (1998) and Qaqish et al. (2003) such associations were not observed $[17,18]$. Thus, in some areas, based on the culture and social criteria, men may cover the highest rate of CE infection [19-21].

In our study, the majority of CE cases were $\geq 50$ years old. This is mainly because CE is a slow-growing cyst in humans and usually presents the signs and symptoms at a higher age of life, whereas the infection may occur in younger people or even teenagers. The fact that all age groups, including children and young adults, were well represented in the confirmed CE cases suggests that both adults and children are susceptible to the infection, as also shown in previous studies [5, 22]. In keeping with global evidence, CE in Iran has no privilege for age. In consistent with our findings, a study by Moldovan which reviewed a total of $190 \mathrm{CE}$ cases during a 7-year period in two counties of Romania reported the highest affected age group to be 60-69 years [23]. However, the disease is not uncommon in young children as $50(10.16 \%)$ of cases in our study were children under 10 years old. In keeping with our findings, a retrospective study regarding $\mathrm{CE}$ cases in children in one of the hospitals in the south of Iran reported 57 children with CE during a 12-year period, 2003-2014 [22].

The present study also revealed that hydatid cyst occurs predominantly in the liver and lung and occasionally in the heart, spleen, abdomen, and pelvis. This is enlightened by the fact that the migrating Echinococcus oncosphere (hexacanth embryo) first encounters the great capillary sites possessed by the 
livers and lungs by which adopt the primarily negotiate hepatic and portal vein route and also the pulmonary filtering system sequentially before any other peripheral organ is involved. Moreover, the lungs are predominantly infected with hydatid cyst than any other organ possibly due to the presence of greater capillary beds in the lungs rather than other organs [24-26].

Generally, retrospective hospital surveys have been criticized for not providing precise estimates of disease incidence as not all hospitals in a particular region or district are included in the study. Besides, retrospective hospital survey data on human CE cannot give an accurate picture of the prevalence of infection. A certain number of cases are not observed in hospitals since the infection is asymptomatic or does not need surgical intervention, and also some data are not available in the hospital records. Yet, despite such limitations, accurate inspection of hospital records offers a useful indication of infection stated as an annual rate of hospital cases. It can indicate the public health importance of the disease and, when done constantly over many years, detects regional variations in the incidence of the infection [27]. It is also worth mentioning that there is a tendency by most surgeons to manage human hydatidosis by exploit a kind of "watch and wait" policy or chemotherapy instead of surgery and only some complicated cases undergoing operations. This again results in a decreased number of patients who undergo surgeries and decrease the number of hospital records.

\section{Conclusion}

Taken together, findings of the current study revealed that human CE is a common infectious disease in the southwest of Iran with a relatively constant rate during the last 15-year period of evaluation. A health education program to increase awareness of how $C E$ is transmitted, along with regular surveillance of the disease would help to reduce the infection rate in this area of Iran.

\section{Declarations}

\section{Acknowledgments}

The technical assistant of staff in Nemazi and Faghihi hospitals is acknowledged.

\section{Funding}

The study was financially supported by the office of vice-chancellor for research of Shiraz University of Medical Sciences (Grant No. 94-01-01-9212).

\section{Availability of data and materials}

SPSS data of the participants can be requested from the authors. Please write to the corresponding author if you are interested in such data.

\section{Authors' contributions}


$B S$, and RS designed the study. RS, AE, ME, MR and HT collected the data. BS and RS carried out the statistical analysis and helped with manuscript writing. RS and AE drafted the manuscript. BS revised and proofread the manuscript. All authors read and approved the final version of the manuscript.

\section{Competing interests}

The authors declare that they have no competing interests.

\section{Consent for publication}

Not applicable.

\section{Ethics approval and consent to participate}

The study was approved by Ethics Committee of the Shiraz University of Medical Sciences. The patients' records were anonymized and de-identified prior to analysis. Confidentiality of the details of the subjects was assured.

\section{References}

1. Deplazes P, Rinaldi L, Alvarez Rojas CA, Torgerson PR, Harandi MF, Romig T, Antolova D, Schurer JM, Lahmar S, Cringoli G et al: Global distribution of alveolar and cystic echinococcosis. Adv Parasitol. 2017; 95:315-493.

2. Fasihi Harandi M, Budke CM, Rostami S: The monetary burden of cystic echinococcosis in Iran. PLoS Negl Trop Dis. 2012; 6(11):e1915.

3. Moshfe A, Sarkari B, Arefkhah N, Nikbakht R, Shahriarirad R, Rezaei Z, Jamshidi A, Moradian F. Seroepidemiological study of cystic echinococcosis in nomadic communities in the southwest of Iran: A population-based study. J Immunoassay Immunochem. 2019;40(2):183-192.

4. Sarkari B, Arefkhah N, Ghorbani F, Meskini F, Yektaeian N, Shahriarirad S, Mostafavi-Pour Z: Seroprevalence of Cystic Echinococcosis and related risk factors for infection among children in a rural community in Fars Province, Southern Iran. Clin Epidemiol Global Health. 2019; [In press].

5. Sarkari B, Sadjjadi SM, Beheshtian MM, Aghaee M, Sedaghat F: Human cystic echinococcosis in Yasuj District in Southwest of Iran: an epidemiological study of seroprevalence and surgical cases over a ten-year period. Zoonoses Public Health. 2010; 57(2):146-150.

6. Khalkhali HR, Foroutan M, Khademvatan S, Majidiani H, Aryamand S, Khezri P, Aminpour A: Prevalence of cystic echinococcosis in Iran: a systematic review and meta-analysis. J 2018; 92(3):260-268. 
7. Ghabouli Mehrabani N, Kousha A, Khalili M, Mahami Oskouei M, Mohammadzadeh M, Alizadeh S, Maleksabet A, Hamidi F: Hydatid Cyst Surgeries in Patients Referred to Hospitals in East Azerbaijan Province during 2009-2011. Iran J Parasitol. 2014; 9(2):233-238.

8. Ahmadi N, Hamidi M: A retrospective analysis of human cystic echinococcosis in Hamedan province, an endemic region of Iran. Ann Trop Med Parasitol. 2008; 102(7):603-609.

9. Hajipirloo HM, Bozorgomid A, Alinia T, Tappeh KH, Mahmodlou R: Human cystic echinococcosis in west azerbaijan, northwest iran: a retrospective hospital based survey from 2000 to 2009. Iranian J Parasitol. 2013; 8(2):323-6.

10. Sadjjadi SM: Present situation of echinococcosis in the Middle East and Arabic North Africa. Parasitol Int. 2006; 55 Suppl:S197-202.

11. MB R: Echinococcosis /hydatidosis in Iran. Iranian J Parasitol. 2009; 4:1-16.

12. McManus DP: Echinococcosis with particular reference to Southeast Asia. Adv Parasitol. 2010; 72:267-303.

13. Abdulhameed MF, Habib I, Al-Azizz SA, Robertson I: A retrospective study of human cystic echinococcosis in Basrah province, Iraq. Acta Trop. 2018; 178:130-133.

14. Budke CM, Carabin H, Ndimubanzi PC, Nguyen H, Rainwater E, Dickey M, Bhattarai R, Zeziulin O, Qian MB. A systematic review of the literature on cystic echinococcosis frequency worldwide and its associated clinical manifestations. Am J Trop Med Hyg. 2013;88(6):1011-27.

15. O'Leary P: A five-year review of human hydatid cyst disease in Turkana District, Kenya. East African Med J. 1976; 53(9):540-544.

16. Aboudaya M: Prevalence of human hydatidosis in Tripoli region of Libya. Int J Zoonoses. 1985; 12(4):304-307.

17. Qaqish A, Nasrieh M, Al-Qaoud K, Craig P, Abdel-Hafez S: The seroprevalences of cystic echinococcosis, and the associated risk factors, in rural-agricultural, bedouin and semi-bedouin communities in Jordan. Ann Trop Med Parasitol. 2003; 97(5):511-520.

18. Cohen H, Paolillo E, Bonifacino R, Botta B, Parada L, Cabrera P, Snowden K, Gasser R, Tessier R, Dibarboure L: Human cystic echinococcosis in a Uruguayan community: a sonographic, serologic, and epidemiologic study. Am J Trop Med Hyg. 1998; 59(4):620-627.

19. Mamishi S, Sagheb S, Pourakbari B: Hydatid disease in Iranian children. J Microbiol Immunol Infect. 2007; 40(5): 428-431

20. Sadeghian H, Sian N: Hydatid Cyst in Children. Pejuhandeh. 2000; 1(5):359-364.

21. Kandeel A, Ahmed E, Helmy H, El Setouhy M, Craig P, Ramzy R: A retrospective hospital study of human cystic echinococcosis in Egypt. East Mediterr Health J. 2004; 10(3):349-357.

22. Sanaei Dashti A, Kadivar MR, Alborzi A, Sadeghi E, Pouladfar GR, Bagherian N, Honar N, Khalifeh M: Analysis of hospital records of children with hydatid cyst in south of Iran. J Parasit Dis. 2017; 41(4):1044-1048. 
23. Moldovan R, Neghina AM, Calma CL, Marincu I, Neghina R: Human cystic echinococcosis in two south-western and central-western Romanian counties: a 7-year epidemiological and clinical overview. Acta Trop. 2012; 121(1):26-29.

24. Kebede W, Hagos A, Girma Z, Lobago F: Echinococcosis/hydatidosis: its prevalence, economic and public health significance in Tigray region, North Ethiopia. Trop Anim Health Prod. 2009; 41(6):865871.

25. Farthing $M$, Jeffries $D$, Anderson J: Infectious diseases, tropical medicine and sexually transmitted diseases. Clin Med. 1994; 4:96-97.

26. Dziri C: Hydatid disease-continuing serious public health problem: introduction. World J 2001; 25(1):1-3.

27. Schantz P, F.L Andersen, H Ouhelli, M Kachani (Eds.): Sources and uses of surveillance data for cystic echinococcosis. Compendium on cystic echinococcosis in Africa and in Middle Eastem countries with special reference to Morocco, Brigham Young University.Provo .1997; pp. 72-84

\section{Table}

Table 2: Demographic features of the CE patients along with the cyst features in Fars, Southwest Iran 


\section{Characteristics}

\section{Frequency (No.)}

Gender

Male

242

48.6

Female

256

51.4

Province

Fars

Kohgiluyeh and Boyer-Ahmad

381

76

Bushehr

35

22

4.4

Khuzestan

14

2.8

Foreign countries

Sistan and Balochistan

Kerman

Kermanshah

Khorasan Razavi

Other Provinces*

$\begin{array}{ll}6 & 1.2 \\ 4 & 0.8 \\ 3 & 0.6 \\ 2 & 0.4 \\ 2 & 0.4 \\ 7 & 1.4\end{array}$

Age groups (years)

$<10$

$10-19$

50

70

10.1

$20-29$

97

14.1

$30-39$

94

19.5

$40-49$

55

18.9

$>50$

Number of cysts

Single

Two cysts

Three cysts

Four cysts

Five or more cysts

Size of cysts $(\mathrm{cm})$

$=<5 \mathrm{~cm}$

6-10

11-15

$>=16$

11

Area of the cyst $\left(\mathrm{cm}^{2}\right)$

$=<25$

26-50

51-75

76-100

$>=100$

389

109

27

8

23

69

164

232

23

179

112

48

62

61
26.4

70

19.6

4.9

1.4

4.2

14.1

33.6

47.5

4.7

38.7

24.2

10.4

13.4

13.2

* Other Provinces: Zanjan, Qom, Lorestan, Isfahan, Ilam, Hormozgan, East Azerbaijan 
Figures

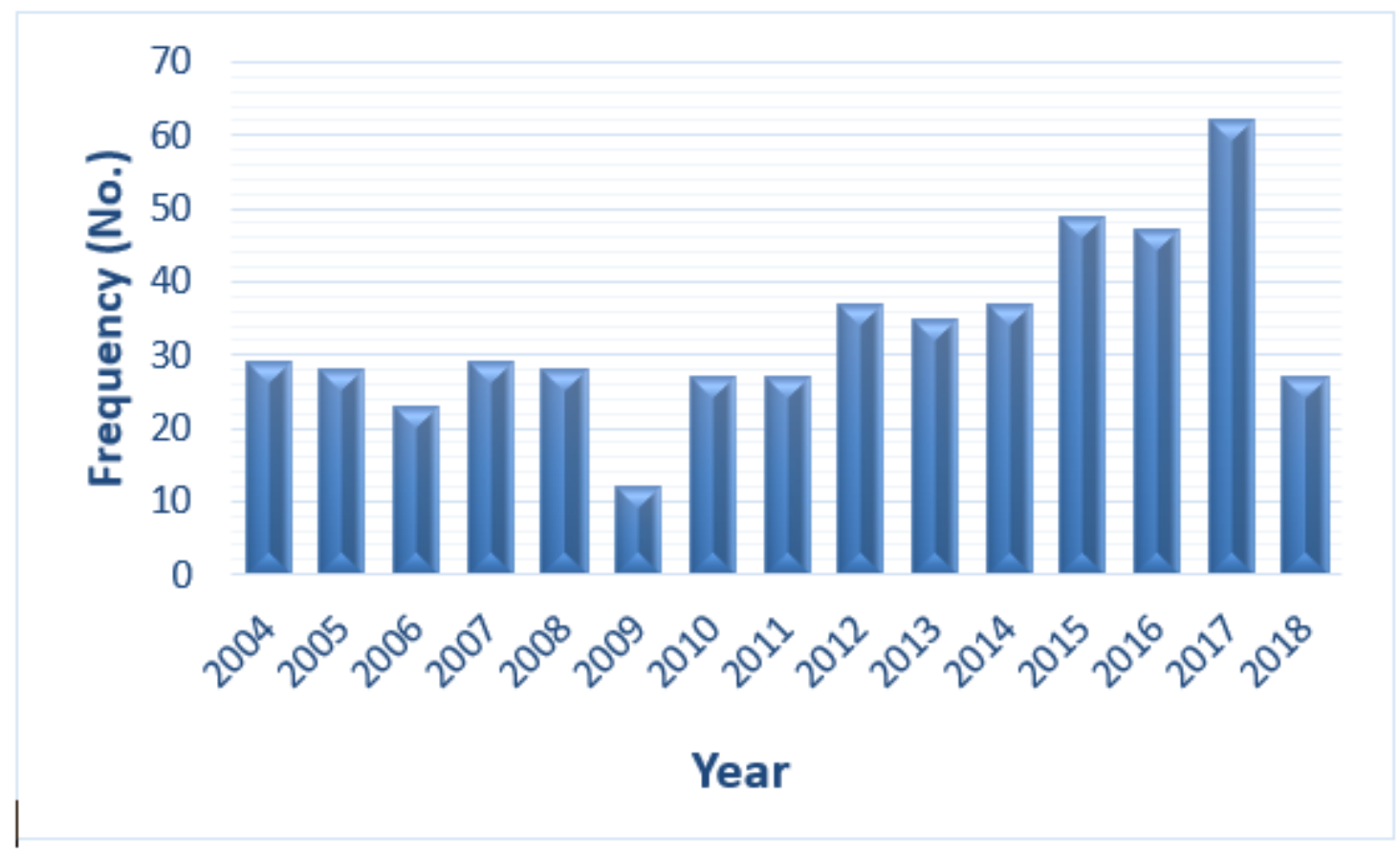

Figure 1

Frequency of CE cases per year, during 2004-2018 in Fars province, Southwest Iran 